\title{
The Local Spectra of Line Graphs
}

\author{
M. A. Fiol ${ }^{1,2}$ \\ Departament de Matemàtica Aplicada IV \\ Universitat Politècnica de Catalunya \\ Barcelona, Spain \\ M. Mitjana ${ }^{1,3}$ \\ Departament de Matemàtica Aplicada I \\ Universitat Politècnica de Catalunya \\ Barcelona, Spain
}

\begin{abstract}
The local spectrum of a graph $G=(V, E)$, constituted by the standard eigenvalues of $G$ and their local multiplicities, plays a similar role as the global spectrum when the graph is "seen" from a given vertex. Thus, for each vertex $i \in V$, the $i$-local multiplicities of all the eigenvalues add up to 1 ; whereas the multiplicity of each eigenvalue $\lambda_{l} \in \operatorname{ev} G$ is the sum, extended to all vertices, of its local multiplicities.

In this work, using the interpretation of an eigenvector as a charge distribution on the vertices, we compute the local spectrum of the line graph $L G$ in terms of the local spectrum of the (regular o semiregular) graph $G$ it derives from. Furthermore, some applications of this result are derived as, for instance, some results related to the number of cycles.
\end{abstract}

Keywords: Graph spectra, eigenvalue, local multiplicity, line graph 


\section{Preliminaries}

Throughout the paper, $G=(V, E)$ denotes a simple connected graph with order $n=|V|$ and size $m=|E|$. We label the vertices with the integers $1,2, \ldots, n$. If $i$ is adjacent to $j$; that is, $(i, j) \in A$, we sometimes write $i \sim j$. The distance between two vertices is denoted by $\operatorname{dist}(i, j)$. The set of vertices which are $k$-appart from vertex $i$ : is $\Gamma_{k}(i)=\{j: \operatorname{dist}(i, j)=k\}$. Thus, the degree of vertex $i$ is just $\delta_{i}=\left|\Gamma_{1}(i)\right| \equiv|\Gamma(i)|$. The eccentricity of a vertex is $\operatorname{ecc}(i):=\max _{1 \leq j \leq n} \operatorname{dist}(i, j)$ and the diameter of the graph is $D=D(G):=\max _{1 \leq i \leq n} \operatorname{ecc}(i)$. Whenever $\operatorname{ecc}(i)=D$, we say that $i$ is a diametral vertex, and also that a pair of vertices $i, j$ such that $\operatorname{dist}(i, j)=D$ is a diametral pair. Moreover, any shortest path between $i$ and $j$ is a diametral path of the graph. The graph is called diametral when all its vertices are diametral.

\section{Some algebraic-graph concepts}

Let us now recall some algebraic graph concepts and results. The adjacency matrix of a graph $G$, denoted by $\boldsymbol{A}=\left(a_{i j}\right)=\boldsymbol{A}(G)$, has entries $a_{i j}=1$ if $i \sim j$ and $a_{i j}=0$ otherwise. Then, the characteristic polynomial of $G$ is just the characteristic polynomial of $\boldsymbol{A}$ :

$$
\phi_{G}(x):=\operatorname{det}(x \boldsymbol{I}-\boldsymbol{A})=\prod_{l=0}^{d}\left(x-\lambda_{l}\right)^{m_{l}} .
$$

Its roots, or eigenvalues of $\boldsymbol{A}$, constitute the spectrum of $G$, denoted by

$$
\operatorname{sp} G:=\operatorname{sp} \boldsymbol{A}=\left\{\lambda_{0}^{m_{0}}, \lambda_{1}^{m_{1}}, \ldots, \lambda_{d}^{m_{d}}\right\}
$$

where the supra-indexes denote multiplicities. The (set of) different eigenvalues of $G$ are represented by

$$
\text { ev } G:=\left\{\lambda_{0}>\lambda_{1}>\cdots>\lambda_{d}\right\} .
$$

It is well known that the diameter of $G$ is lesser than the number of different eigenvalues; that is, $D(G) \leq d$ (see, for instance, Biggs [5]). When $D(G)=d$ we say that $G$ is an extremal graph.

\footnotetext{
1 This research was supported by the Secretaria de Estado de Universidades e Investigación (Ministerio de Educación y Ciencia), Spain, and the European Regional Development Fund (ERDF) under project TEC2005-03575.

2 Email: fiol@ma4.upc.edu

3 Email: margarida.mitjana@upc.edu
} 


\section{The spectral decomposition}

For each eigenvalue $\lambda_{l}, 0 \leq l \leq d$, let $\boldsymbol{U}_{l}$ be the matrix whose columns form an orthonormal basis for the $\lambda_{l}$-eigenspace $\mathcal{E}_{l}:=\operatorname{Ker}\left(\boldsymbol{A}-\lambda_{l} \boldsymbol{I}\right)$. The (principal) idempotents of $\boldsymbol{A}$ are the matrices $\boldsymbol{E}_{l}:=\boldsymbol{U}_{l} \boldsymbol{U}_{l}^{\top}$ representing the orthogonal projections onto $\mathcal{E}_{l}$. Thus, in particular, $\boldsymbol{E}_{0}=\frac{1}{\|\boldsymbol{v}\|^{2}} \boldsymbol{v} \boldsymbol{v}^{\top}$, where $\boldsymbol{v}=\left(v_{1}, v_{2}, \ldots, v_{n}\right)^{\top}$ denotes the normalized positive eigenvector. From their structure, it is readily checked that such matrices satisfy the following properties (see, for instance, Godsil [25]):

(a.1) $\boldsymbol{E}_{l} \boldsymbol{E}_{h}= \begin{cases}\boldsymbol{E}_{l} & \text { if } l=h \\ \mathbf{0} & \text { otherwise; }\end{cases}$

(a.2) $\boldsymbol{A} \boldsymbol{E}_{l}=\lambda_{l} \boldsymbol{E}_{l}$;

(a.3) $p(\boldsymbol{A})=\sum_{l=0}^{d} p\left(\lambda_{l}\right) \boldsymbol{E}_{l}$, for any polynomial $p \in \mathbb{R}[x]$.

In particular, notice that if, in (a.3), we take $p=1$ and $p=x$ we obtain, respectively, $\sum_{l=0}^{d} \boldsymbol{E}_{l}=\boldsymbol{I}$ (as expected, since the sum of all orthogonal projections gives the original vector), and the so-called "Spectral Decomposition Theorem" $\sum_{l=0}^{d} \lambda_{l} \boldsymbol{E}_{l}=\boldsymbol{A}$. The following spectral decompositions of the canonical vectors are repeatedly used below:

$$
\boldsymbol{e}_{i}=\boldsymbol{z}_{i 0}+\boldsymbol{z}_{i 1}+\cdots+\boldsymbol{z}_{i d}=\boldsymbol{z}_{i 0}+\boldsymbol{z}_{i} \quad(1 \leq i \leq n)
$$

where $\boldsymbol{z}_{i l}:=\boldsymbol{E}_{l} \boldsymbol{e}_{i} \in \mathcal{E}_{l}, 0 \leq l \leq d$, and $\boldsymbol{z}_{i} \in \mathcal{E}_{1} \oplus \cdots \oplus \mathcal{E}_{d}=\boldsymbol{v}^{\perp}$. Moreover,

$$
\boldsymbol{z}_{i 0}=\frac{\left\langle\boldsymbol{e}_{i}, \boldsymbol{v}\right\rangle}{\|\boldsymbol{v}\|^{2}} \boldsymbol{v}=\frac{v_{i}}{\|\boldsymbol{v}\|^{2}} \boldsymbol{v}
$$

In particular, for regular graphs, $\boldsymbol{z}_{i 0}=(1 / n) \boldsymbol{j}$.

\section{The local multiplicity}

Given two vertices $i, j$ and any eigenvalue $\lambda_{l}$, Garriga, Yebra and the first author in [16], introduced the concept of crossed (ij-)local multiplicity of $\lambda_{l}$ as $m_{i j}\left(\lambda_{l}\right):=\left\langle\boldsymbol{z}_{i l}, \boldsymbol{z}_{j l}\right\rangle$. Note that this corresponds to the $i j$-entry of the idempotent $\boldsymbol{E}_{l}$ since, using the symmetric character of $\boldsymbol{E}_{l}$ and property (a.1),

$$
\left\langle\boldsymbol{z}_{i l}, \boldsymbol{z}_{j l}\right\rangle=\left\langle\boldsymbol{E}_{l} \boldsymbol{e}_{i}, \boldsymbol{E}_{l} \boldsymbol{e}_{j}\right\rangle=\left\langle\boldsymbol{E}_{l} \boldsymbol{e}_{i}, \boldsymbol{e}_{j}\right\rangle=\left(\boldsymbol{E}_{l}\right)_{i j} .
$$

From the above properties of the idempotents we have that the crossed local multiplicities satisfy the following: 
(b.1) $\sum_{l=0}^{d} m_{i j}\left(\lambda_{l}\right)= \begin{cases}1 & \text { if } i=j ; \\ 0 & \text { otherwise. }\end{cases}$

(b.2) $\sum_{j \sim i} m_{i j}\left(\lambda_{l}\right)=\lambda_{l} m_{i i}\left(\lambda_{l}\right)$;

(b.3) $a_{i j}^{k}=\sum_{l=0}^{d} m_{i j}\left(\lambda_{l}\right) \lambda_{l}^{k}$,

where $a_{i j}^{k}:=\left(\boldsymbol{A}^{k}\right)_{i j}$ is the number of $k$-walks between vertices $i$ and $j$ (see Godsil $[23,24]$ ) including closed walks (when $i=j$ ). Under some assumptions, the local crossed multiplicities admit closed expressions. For instance, when $\lambda=\lambda_{0}$, we have

$$
m_{i j}\left(\lambda_{0}\right)=\left\langle\frac{v_{i}}{\|\boldsymbol{v}\|^{2}} \boldsymbol{v}, \frac{v_{j}}{\|\boldsymbol{v}\|^{2}} \boldsymbol{v}\right\rangle=\frac{v_{i} v_{j}}{\|\boldsymbol{v}\|^{2}} .
$$

Another example is given by the next result, see [16].

Lemma 1.1 Let $i, j$ be a pair of diametral vertices of an extremal graph $G$ with normalized positive eigenvector $\boldsymbol{v}$. Then, the number of diametral paths between them and the crossed ij-local multiplicities are respectively given by

$$
\begin{aligned}
& \qquad a_{i j}^{d}=\pi_{0} \frac{v_{i} v_{j}}{\|\boldsymbol{v}\|^{2}}, \quad m_{i j}\left(\lambda_{l}\right)=(-1)^{l} \frac{\pi_{0}}{\pi_{l}} \frac{v_{i} v_{j}}{\|\boldsymbol{v}\|^{2}} \quad(1 \leq l \leq d), \\
& \text { where } \pi_{l}:=\prod_{h=0, h \neq l}^{d}\left|\lambda_{l}-\lambda_{h}\right| \quad(0 \leq l \leq d) .
\end{aligned}
$$

Notice that for regular graphs, from the above lemma and $a_{i j}^{k}=0$ for any $k \leq d-1$, property (b.3) yields:

$$
\sum_{l=0}^{d} \frac{(-1)^{l}}{\pi_{l}} \lambda_{l}^{k}=0 \quad(0 \leq k \leq d-1) ; \quad \sum_{l=0}^{d} \frac{(-1)^{l}}{\pi_{d}} \lambda_{l}^{d}=1 .
$$

as we already knew.

\section{The local spectrum}

The crossed $i j$-local multiplicities seem to have a special relevance when $i=j$. In this case $m_{i i}\left(\lambda_{l}\right)=\left\|\boldsymbol{z}_{i l}\right\|^{2} \geq 0$, denoted also by $m_{i}\left(\lambda_{l}\right)$, is referred to as the $i$-local multiplicity of $\lambda_{l}$. (In particular, (2) yields $m_{i}\left(\lambda_{0}\right)=v_{i}^{2} /\|\boldsymbol{v}\|^{2}$.) In [15] it was noted that when the graph is "seen" from vertex $i$, the $i$-local multiplicities play a similar role as the standard multiplicities, so justifying the name. Indeed, by property (b.1) note that, for each vertex $i$, the $i$-local multiplicities of all the eigenvalues add up to $1: \sum_{l=0}^{d} m_{i}\left(\lambda_{l}\right)=1$ whereas the 
multiplicity of each eigenvalue $\lambda_{l}$ is the sum, extended to all vertices, of its local multiplicities since

$$
m\left(\lambda_{l}\right)=\operatorname{tr} \boldsymbol{E}_{l}=\sum_{i=1}^{n} m_{i i}\left(\lambda_{l}\right) .
$$

Moreover, property (b.3) tells us that the number of closed walks of length $k$ going through vertex $i, a_{i j}^{k}$, can be computed in a similar way as is computed the whole number of such walks in $G$ by using the "global" multiplicities. Some closely related parameters are the Cvetković's "angles" of $G$, which are defined as the cosines $\cos \beta_{i l}, 1 \leq i \leq n, 0 \leq l \leq d$, with $\beta_{i l}$ being the angle between $\boldsymbol{e}_{i}$ and the eigenspace $\operatorname{Ker}\left(\boldsymbol{A}-\lambda_{l} \boldsymbol{I}\right)$ (notice that $m_{i}\left(\lambda_{l}\right)=\cos ^{2} \beta_{i l}$.) For a number of applications of these parameters, see for instance Cvetković, Rowlinson, and Simić [11].

By considering only the eigenvalues, say $\mu_{0}\left(=\lambda_{0}\right)>\mu_{1}>\cdots>\mu_{d_{i}}$, with non-null local multiplicities, we can now define the (i-)local spectrum as

$$
\operatorname{sp}_{i} G:=\left\{\lambda^{m_{i}\left(\lambda_{0}\right)}, \mu_{1}^{m_{i}\left(\mu_{1}\right)}, \ldots, \mu_{d_{i}}^{m_{i}\left(\mu_{d_{i}}\right)}\right\}
$$

with (i-)local mesh, or set of distinct eigenvalues, $\mathcal{M}_{i}:=\left\{\lambda_{0}>\mu_{1}>\cdots>\right.$ $\left.\mu_{d_{i}}\right\}$. Then it can be proved that the eccentricity of $i$ satisfies a similar upper bound as that satisfied by the diameter of $G$ in terms of its distinct eigenvalues. More precisely, $\operatorname{ecc}(i) \leq d_{i}=\left|\mathcal{M}_{i}\right|-1$ (see [16].)

From the $i$-local spectrum (5), it is natural to consider the analogous function of the characteristic polynomial, which we call the $i$-local characteristic function, defined as:

$$
\phi_{i}(x):=\prod_{l=0}^{d_{i}}\left(x-\mu_{l}\right)^{m_{i}\left(\mu_{l}\right)} .
$$

As expected, such a function can be computed from the knowledge of the characteristics polynomials of $G$ and $G \backslash i$.

Proposition 1.2 Given a vertex $i$ of a graph $G$, its $i$-local characteristic function is

$$
\phi_{i}(x)=e^{\int \phi_{G \backslash i}(x) / \phi_{G}(x) d x} .
$$

Proof. First note that the characteristic polynomial $\phi_{G \backslash i}(x)$ is just the $i i$ entry of the adjoint matrix of $x \boldsymbol{I}-\boldsymbol{A}[9]$ which, in turn, can be written as 


$$
\begin{aligned}
\operatorname{det}(x \boldsymbol{I}-\boldsymbol{A})(x \boldsymbol{I}-\boldsymbol{A})^{-1} & =\phi_{G}(x)(x \boldsymbol{I}-\boldsymbol{A})^{-1} \\
& =\phi_{G}(x) \sum_{l=0}^{d} \frac{1}{x-\lambda_{l}} \boldsymbol{E}_{l},
\end{aligned}
$$

where we have used property (a.3) extended to the continuity points of any rational function (in our case, $x \neq \lambda_{l}$ ). Hence,

$$
\phi_{G \backslash i}(x)=\phi_{G}(x) \sum_{l=0}^{d} \frac{m_{i}\left(\lambda_{l}\right)}{x-\lambda_{l}} .
$$

and, thus,

$$
\frac{\phi_{G \backslash i}(x)}{\phi_{G}(x)}=\sum_{l=0}^{d} \frac{m_{i}\left(\lambda_{l}\right)}{x-\lambda_{l}}=\sum_{l=0}^{d_{i}} \frac{m_{i}\left(\mu_{l}\right)}{x-\mu_{l}}=\frac{\phi_{i}^{\prime}(x)}{\phi_{i}(x)} .
$$

Then, we obtain the claimed result integrating both sides with respect to $x$ and isolating $\phi_{i}(x)$.

As a by-product, note also that, from (8) and adding over all the vertices, we get the known result

$$
\sum_{i=1}^{n} \phi_{G \backslash i}(x)=\phi_{G}(x) \sum_{l=0}^{d} \sum_{i=1}^{n} \frac{m_{i}\left(\lambda_{l}\right)}{x-\lambda_{l}}=\phi_{G}(x) \sum_{l=0}^{d} \frac{m_{l}}{x-\lambda_{l}}=\phi_{G}^{\prime}(x) .
$$

(See, for instance, [25].)

\section{Eigenvectors and Patterns}

In this section we first recall the simple interpretation of eigenvalues and eigenvectors in terms of charge displacement. Second, some easy but very useful results based on this approach are discussed.

\section{Eigenvectors in a graph}

As commented above, a very simple, yet surprisingly useful, idea is the interpretation of the eigenvectors and eigenvalues of a graph as a dynamic process of "charge displacement" (see, for instance, Godsil [25]). To this end, suppose that $\boldsymbol{A}$ is the adjacency matrix of a graph $G=(V, E)$ and $\boldsymbol{v}$ a right eigenvector of $\boldsymbol{A}$ with eigenvalue $\lambda$. If we think $\boldsymbol{v}$ as a function from $V$ to the complex numbers, we associate $v_{i}$ to the "initial charge" (or weight) of vertex 
$i$. Since $\boldsymbol{A}$ is a $0-1$ matrix, the equation $\boldsymbol{A} \boldsymbol{v}=\lambda \boldsymbol{v}$ is equivalent to

$$
(\boldsymbol{A} \boldsymbol{v})_{i}=\sum_{j=1}^{n} a_{i j} v_{j}=\sum_{j \sim i} v_{j}=\lambda v_{i} \quad \text { for all } i \in V .
$$

Thus, the sum of the charges of the neighbors of $i$ is $\lambda$ times the charge of vertex $i$. In [25] it is shown how this idea can be extended to "vector charges", so leading to the important area of research in graph theory known as representation theory.

\section{The spectra of line graphs}

The line graph $L G$ of any graph $G=(V, E)$ is defined as follows. Each vertex in $L G$ represents an edge of $G, V(L G)=\{i j:(i, j) \in E\}$, and two vertices of $L G$ are adjacent whenever the corresponding edges in $G$ have a vertex in common.

Since the classical paper of Sachs [31], the spectra of line graphs have been studied extensively. In [19] the authors used the mentioned idea of interpreting the eigenvectors as a certain charge distributions to prove that, if a $\delta$-regular graph $G$ has the eigenvector $\boldsymbol{u}$ with eigenvalue $\lambda \neq-\delta$, then the vector $\boldsymbol{v}$ with entries that $v_{i j}=u_{i}+u_{j}$, where $(i, j) \in E$, is a $(\lambda+\delta-2)$-eigenvector of $L G$. Let us now see that the method can be used to derive the local spectrum of $L G$.

\section{The local spectrum of a regular line graph}

The following result tells us how to compute the local spectrum of a line graph from the local spectrum of the (regular) graph it derives from.

Theorem 3.1 Let $G$ be a $\delta$-regular graph with eigenvalue $\lambda$, multiplicity $m(\lambda)$, and (crossed) local multiplicities $m_{i j}=m_{i j}(\lambda), i, j \in V$. Then, the crossed local multiplicities of $\lambda^{\prime}=\lambda+\delta-2$ in the line graph $L G$, with vertices denoted in the same way as the edges of $G$; that is, $(i, j)$, are given by the expressions:

$$
\begin{aligned}
m_{(i, j)(k, h)}\left(\lambda^{\prime}\right) & =\frac{m_{i k}(\lambda)+m_{i h}(\lambda)+m_{j k}(\lambda)+m_{j h}(\lambda)}{\delta+\lambda} \quad(\lambda \neq-\delta), \\
m_{(i, j)(k, h)}(-2) & =\alpha-\sum_{\lambda \neq-\delta} m_{(i, j)}(\lambda),
\end{aligned}
$$

where $\alpha=0$ if $(i, j) \neq(k, h)$ and $\alpha=1$ otherwise. 
Proof. Assume first that $\lambda \neq-\delta$, and let $U$ be the set of $m(\lambda)$ column vectors of the matrix $\boldsymbol{U}$ (recall that these vectors constitute an orthonormal basis of the corresponding eigenspace $\mathcal{E}$ ). Then, given $\boldsymbol{u} \in U$, we already know that the vector $\boldsymbol{v}$ with components $v_{(i, j)}=u_{i}+u_{j}$ is a $\lambda^{\prime}(=\lambda+\delta-2)$-eigenvector of $L G$. Notice that, since

$$
\sum_{(i, j) \in E}\left(u_{i}+u_{j}\right)^{2}=\sum_{i \in V} \delta u_{i}^{2}+\sum_{(i, j) \in E} 2 u_{i} u_{j}=\delta+\langle\boldsymbol{u}, \boldsymbol{A} \boldsymbol{u}\rangle=\delta+\lambda,
$$

the corresponding normalized vector has components $\frac{u_{i}+u_{j}}{\sqrt{\delta+\lambda}}$. Then, the crossed local multiplicity, at vertex $(i, j)$, of $\lambda^{\prime}$ is

$$
\begin{aligned}
m_{(i, j)(k, h)}\left(\lambda^{\prime}\right) & =\sum_{\boldsymbol{u} \in U} \frac{\left(u_{i}+u_{j}\right)\left(u_{k}+u_{h}\right)}{\delta+\lambda} \\
& =\frac{1}{\delta+\lambda} \sum_{\boldsymbol{u} \in U}\left(u_{i} u_{h}+u_{i} u_{k}+u_{j} u_{k}+u_{j} u_{h}\right) \\
& =\frac{m_{i h}(\lambda)+m_{i k}(\lambda)+m_{j k}(\lambda)+m_{j h}(\lambda)}{\delta+\lambda} .
\end{aligned}
$$

Finally, the crossed local multiplicity of the eigenvalue $\lambda^{\prime}=-2$ is obtained by using the formula (b.1).

Notice that, in particular, the local multiplicities of $\lambda^{\prime}$ are $m_{(i, j)}\left(\lambda^{\prime}\right)=$ $m_{(i, j)(i, j)}\left(\lambda^{\prime}\right)$, which gives:

$$
\begin{aligned}
m_{(i, j)}(\lambda+\delta-2) & =\frac{m_{i}(\lambda)+2 m_{i j}(\lambda)+m_{j}(\lambda)}{\delta+\lambda} \quad\left(\lambda^{\prime} \neq-2\right) \\
m_{(i, j)}(-2) & =1-\sum_{\lambda \neq-2} \frac{m_{i}(\lambda)+2 m_{i j}(\lambda)+m_{j}(\lambda)}{\delta+\lambda}
\end{aligned}
$$

Then, as expected,

$$
\begin{aligned}
\sum_{(i, j) \in E} m_{(i, j)}\left(\lambda^{\prime}\right) & =\frac{1}{\delta+\lambda} \sum_{i \sim j}\left(m_{i}(\lambda)+2 m_{i j}(\lambda)+m_{j}(\lambda)\right)= \\
& =\frac{1}{\delta+\lambda}\left(\sum_{i \in V} \delta m_{i}(\lambda)+\sum_{(i, j) \in E} m_{i j}(\lambda)\right)= \\
& =\frac{1}{\delta+\lambda} \sum_{i \in V}(\delta+\lambda) m_{i}(\lambda)=m(\lambda) .
\end{aligned}
$$

where the last two equalities come from property (b.2) and equality (4), respectively. 
Let us use the above relationship between the local multiplicities of a graph and its line graph to compute the number of $k$-circuits in the line graph $L G$ based on vertex $(i, j)$, this is the number of walks of length $k$ between vertices $i, j$ in the graph $G$. Again, vertices in $L G$ are denoted in the same way as the edges of $G$; that is, $(i, j)$.

Proposition 3.2 Let $G$ be a $\delta$-regular graph with spectrum

$$
\operatorname{sp} G=\left\{\lambda_{0}^{m_{0}}, \lambda_{1}^{m_{1}}, \ldots, \lambda_{d}^{m_{d}}\right\}
$$

and crossed (ij)-local multiplicities $m_{i j}\left(\lambda_{l}\right), i, j \in V \quad 0 \leq l \leq d$. Then, the number of circuits of length $k$ through vertex $(i, j)$ in the line graph $L G$, is given by

$$
\left(A_{L G}^{k}\right)_{(i, j)(i, j)}=(-2)^{k}+\sum_{\lambda_{l} \neq-\delta}^{d-1} \sum_{p=0}^{k-1} K_{p} \lambda_{l}^{k-p-1}\left(m_{i}\left(\lambda_{l}\right)+m_{j}\left(\lambda_{l}\right)+2 m_{i j}\left(\lambda_{l}\right)\right)
$$

where, for each $k$, the coefficient of $\lambda_{l}^{k-p-1}$ is

$$
K_{p}:=\sum_{r=0}^{p}\left(\begin{array}{l}
k \\
r
\end{array}\right)\left(\begin{array}{c}
k-r-1 \\
p-r
\end{array}\right) \delta^{p-r}(-2)^{r}
$$

Proof.

$$
\begin{aligned}
& \left(A_{L G}^{k}\right)_{(i, j)(i, j)}= \\
= & \sum_{l=0}^{d}\left(\lambda_{l}+\delta-2\right)^{k} \frac{m_{i}+2 m_{i j}+m_{j}}{\lambda_{l}+\delta}= \\
= & \sum_{l=0}^{d-1}\left(\lambda_{l}+\delta-2\right)^{k} \frac{m_{i}+2 m_{i j}+m_{j}}{\lambda_{l}+\delta}+(-2)^{k}\left[1-\sum_{l=0}^{d-1} \frac{m_{i}+2 m_{i j}+m_{j}}{\lambda_{l}+\delta}\right]= \\
= & (-2)^{k}+\sum_{l=0}^{d-1} \frac{\left(\lambda_{l}+\delta-2\right)^{k}-(-2)^{k}}{\lambda_{l}+\delta}\left(m_{i}+2 m_{i j}+m_{j}\right)= \\
= & (-2)^{k}+ \\
& +\sum_{l=0}^{d-1}\left[\left(\begin{array}{c}
k \\
0
\end{array}\right)\left(\lambda_{l}+\delta\right)^{k-1}+\cdots+\left(\begin{array}{c}
k \\
k-1
\end{array}\right)(-2)^{k-1}\right]\left(m_{i}+2 m_{i j}+m_{j}\right)
\end{aligned}
$$

collecting coefficients of $\lambda_{l}^{k-p-1}, 0 \leq p \leq k-1$, we obtain the result. 
In particular for $p=0,1,2$ we have

$K_{0}=1, \quad K_{1}=(k-1) \delta-2 k, \quad K_{2}=\frac{(k-1)(k-2)}{2} \delta^{2}-2 \delta k(k-2)-2 k(k-1)$

From the previous result we may calculate the number of circuits rooted at a given vertex $(i, j)$, in the line graph as a function of the circuits rooted in vertex $i$ and walks that contain edge $(i, j)$ in the original graph $G$.

\section{Corollary 3.3}

$$
\begin{gathered}
\left(A_{L G}^{k}\right)_{(i, j)(i, j)}=(-2)^{k}+2 \sum_{p=0}^{k-1} K_{p}\left(A_{G}\right)_{i i}^{k-p-1}+2 \sum_{p=0}^{k-1} K_{p}\left(A_{G}\right)_{i j}^{k-p-1} \\
\left(A_{L G}^{k}\right)_{(i, j)(i, j)}=(-2)^{k}+\sum_{l=0}^{d-1} \sum_{p=0}^{k-1} K_{p} \lambda_{l}^{k-p-1}\left(m_{i}\left(\lambda_{l}\right)+m_{j}\left(\lambda_{l}\right)+2 m_{i j}\left(\lambda_{l}\right)\right)
\end{gathered}
$$

\section{Local multiplicities in a cycle $C_{n}$}

Let us consider $C_{n}$ the cycle of orden $n$. As the line graph of a cycle $\boldsymbol{C}_{n}$ is itself, $L \boldsymbol{C}_{n}=\boldsymbol{C}_{n}$, the number of $k$-circuits rooted in vertex $i$ in $\boldsymbol{C}_{n}$, or in vertex $(i, j)$ in $L \boldsymbol{C}_{n}$ is equal, this fact allow us to derive simple relations between the crossed and local multiplicities of each eigenvalue.

Taking into account the result in (10), and $\delta=2$

$$
m_{i}\left(\lambda_{l}\right)=m_{(i, j)(i, j)}\left(\lambda_{l}\right)=\frac{m_{i}\left(\lambda_{l}\right)+2 m_{i j}\left(\lambda_{l}\right)+m_{j}\left(\lambda_{l}\right)}{\lambda_{l}+2}
$$

we get the crossed local multiplicity $m_{i j}\left(\lambda_{l}\right)$ correspondig to adjacent vertices $i, j$ in $\boldsymbol{C}_{n}$ and $\lambda_{l} \neq-\delta$

$$
m_{i}\left(\lambda_{l}\right)=\frac{2 m_{i}\left(\lambda_{l}\right)+2 m_{i j}\left(\lambda_{l}\right)}{\lambda_{l}+2} \Rightarrow m_{i j}\left(\lambda_{l}\right)=\frac{\lambda_{l} m_{i}\left(\lambda_{l}\right)}{2}
$$

Case $\lambda_{l}=-\delta$ follows from (11)

$$
m_{i}(-2)=1-2 \sum_{\lambda_{l} \neq-2} \frac{m_{i}\left(\lambda_{l}\right)+m_{i j}\left(\lambda_{l}\right)}{\lambda_{l}+2}
$$

Crossed local multiplicities corresponding to non-adjacent vertices can also be computed.

In order to simplify the notation, for a given eigenvalue $\lambda_{l}$ of the cycle graph $C_{n}$ and vertices $i, j$ with $\operatorname{dist}(i, j)=k$, we will denote the crossed (ij- 
)local multiplicity $m_{k}\left(\lambda_{l}\right):=m_{i j}\left(\lambda_{l}\right)$, or $m_{k}$ for short if there is no confusion about the eigenvalue we are referring to.

Let $p, q, i, j, r, s$ vertices of the cycle graph $C_{n}$, such that $i \sim j$ and $r \sim s$ so that $(i, j)$ and $(r, s)$ are vertices in the line graph $L C_{n}$. Let us suppose $\operatorname{dist}(p, q)=k=\operatorname{dist}((i, j),(r, s)), 0 \leq k<\left\lfloor\frac{n}{2}\right\rfloor$. Then, as above, the equality $m_{p q}\left(\lambda_{l}\right)=m_{(i, j)(k, l)}\left(\lambda_{l}\right)$ and (10) leeds to

$$
m_{k}=\frac{m_{k+1}+2 m_{k}+m_{k-1}}{\lambda_{l}+2}
$$

that gives

$$
\lambda_{l} m_{0}=2 m_{1}
$$

and

$$
\lambda_{l} m_{k}=m_{k-1}+m_{k+1}
$$

$0<k<\left\lfloor\frac{n}{2}\right\rfloor$, or equivalently,

$$
\left(\begin{array}{cc}
\lambda_{l} & -1 \\
1 & 0
\end{array}\right)\left(\begin{array}{c}
m_{k} \\
m_{k-1}
\end{array}\right)=\left(\begin{array}{c}
m_{k+1} \\
m_{k}
\end{array}\right) \Leftrightarrow\left(\begin{array}{cc}
\lambda_{l} & -1 \\
1 & 0
\end{array}\right)^{k}\left(\begin{array}{l}
m_{1} \\
m_{0}
\end{array}\right)=\left(\begin{array}{c}
m_{k+1} \\
m_{k}
\end{array}\right)
$$

With some linear algebra, we can derive explicit expressions for the crossed multiplicities as function of the corresponding local multiplicity. Define $\Phi_{1,2}=$ $\frac{\lambda_{l} \pm \sqrt{\lambda_{l}^{2}-4}}{2}$, then

$$
m_{k+1}=\frac{m_{0}}{\Phi_{1}-\Phi_{2}}\left[\frac{\lambda_{l}}{2}\left(\Phi_{1}^{k+1}-\Phi_{2}^{k+1}\right)+\Phi_{1}^{k}-\Phi_{2}^{k}\right],
$$

$0<k<\left\lfloor\frac{n}{2}\right\rfloor$. In particular we get

$$
m_{1}=\frac{\lambda_{l}}{2} m_{0} ; \quad m_{2}=\frac{\lambda_{l}^{2}-2}{2} m_{0} ; \quad m_{3}=\frac{1}{2} \lambda_{l}\left(\lambda_{l}^{2}-3\right) m_{0} ; \ldots
$$

Crossed local multiplicities corresponding to eigenvalue $\lambda_{l}=-\delta$ can be computed with the previous result and (11).

The local multiplicities of a cycle $C_{n}$ correspondig to a given eigenvalue $\lambda$ satisfy

$$
m_{k}(\lambda)=T_{k}\left(\frac{\lambda}{2}\right) m_{0}
$$

for $0 \leq k \leq\left\lfloor\frac{n}{2}\right\rfloor \leq n$, where $T_{n}(x)$ is the Chebyshev polynomial of degree $n$. 


\subsection{Semiregular graphs(?)}

Here we will see that, as in the case of regular graphs, the eigenvalues of line graphs of semiregular graphs can also be determined. A graph $G=(V, E)$ is called semiregular when, for some integers $\delta_{1}, \delta_{2}$, every edge $\{i, j\} \in E$ has its endvertices with degrees $\delta(i)=\delta_{1}$ and $\delta(j)=\delta_{2}$. In this case, we speak also of a $\left(\delta_{1}, \delta_{2}\right)$-semiregular graph. Of course, the case $\delta_{1}=\delta_{2}$ corresponds to the standard regularity and, otherwise, the graph $G$ must be bipartite, $V=V_{1} \cup V_{2}$, with vertices in each stable set $V_{i}$ having the same degree $\delta_{i}$, $i=1,2$. The following result was proved in [19]:

Theorem 3.4 Let $G$ be a $\left(\delta_{1}, \delta_{2}\right)$-semiregular graph, $\delta_{1}, \delta_{2}>1$, with distinct eigenvalues ev $G=\left\{ \pm \lambda_{l}: 0 \leq l \leq r\right\}$. Then, its line graph $L G$, with adjacency matrix $\boldsymbol{A}_{L}$, has the eigenvalues ev $L G=\left\{-2, \pm \lambda_{l}^{\prime}: 0 \leq l \leq r\right\}$, where

$$
\lambda_{l}^{\prime}=\frac{\delta_{1}+\delta_{2}}{2} \pm \frac{1}{2} \sqrt{\left(\delta_{1}-\delta_{2}\right)^{2}+4 \lambda_{l}^{2}}-2 \quad(0 \leq l \leq r) .
$$

\section{References}

[1] C. Balbuena, D. Ferrero, X. Marcote, and I. Pelayo, Algebraic properties of a digraph and its line digraph, J. Interconnection Networks 4 (2003), no. 4, 377-393.

[2] E. Bannai and T. Ito, Algebraic Combinatorics I: Association Schemes. Benjamin/Cummings, Menlo Park, CA, 1984.

[3] N. Biggs, Intersection matrices for linear graphs, in: Combinatorial Mathematics and its Applications (ed. D.J.A. Welsh), 15-23, Academic Press, London, 1971.

[4] N. Biggs, Some odd graph theory, Ann. New York Acad. Sci. 319 (1979), 71-81.

[5] N. Biggs, Algebraic Graph Theory. Cambridge University Press, Cambridge, 1974; second edition: 1993.

[6] L. Branković, and D. Cvetković, The eigenspace of the eigenvalue -2 in generalized line graphs and a problem in security of statistical databases, Univ. Beograd. Publ. Elektrotehn. Fak. Ser. Mat. 14 (2003), 37-48.

[7] A.E. Brouwer, A.M. Cohen and A. Neumaier, Distance-Regular Graphs. Springer-Verlag, Berlin, 1989.

[8] F. Comellas, M.A. Fiol, J. Gimbert and M. Mitjana, Weakly distance-regular digraphs, J. Combin. Theory Ser. B 90 (2004), no. 2, 233-255. 
[9] D. Cvetković and M. Doob, Developments in the theory of graph spectra, Linear and Multilinear Algebra 18 (1985), 153-181.

[10] D. Cvetković, M. Doob, and and S. Simić, Generalized line graphs, J. Graph Theory 5 (1981), 385-399.

[11] D. Cvetković, P. Rowlinson, and S. Simić, Eigenspaces of Graphs. Cambridge University Press, Cambridge, 1997.

[12] E.R. van Dam, Bounds on special subsets in graphs, eigenvalues and association schemes, J. Algebraic Combin. 7 (1998), no. 3, 321-332.

[13] J. Fàbrega and M.A. Fiol, Maximally connected digraphs, J. Graph Theory 13 (1989), no. 3, 657̂े-668.

[14] M.A. Fiol and E. Garriga, From local adjacency polynomials to locally pseudodistance-regular graphs, J. Combin. Theory Ser. B 71 (1997), 162-183.

[15] M.A. Fiol, E. Garriga, and J.L.A. Yebra, Locally pseudo-distance-regular graphs, J. Combin. Theory Ser. B 68 (1996), 179-205.

[16] M.A. Fiol, E. Garriga, and J.L.A. Yebra, Boundary graphs: The limit case of a spectral property, submitted, Discrete Math. 226 (2001) 155-173.

[17] M.A. Fiol, J. Gimbert and M. Miller, Multipartite Moore digraphs, Linear Algebra Appl., to appear, 2006.

[18] M.A. Fiol and A.S. Lladó, The partial line digraph technique in the design of large interconnection networks, IEEE Trans. Comput. C-41, no. 7, (1992), $848-857$.

[19] M.A. Fiol and M. Mitjana, The spectra of some families of digraphs, Linear Algebra Appl., submitted, 2006.

[20] M.A. Fiol , J.L.A Yebra and I. Alegre, Line digraph iterations in the $(d, k)$ digraph problem, IEEE Trans. Comput. C-33, no. 5, (1984), 400-403.

[21] M.A. Fiol, J.L.A. Yebra and J. Fàbrega, Sequence graphs and interconnection networks, Ars Combin. 16, (1983), 7-14.

[22] E. Garriga, "Contribució a la Teoria Espectral de Grafs. Problemes Mètrics i Distància-Regularitat," Ph.D. Thesis (in Catalan), Universitat Politècnica de Catalunya, Barcelona, 1997.

[23] C.D. Godsil, Graphs, groups, and polytopes, in: Combinatorial Mathematics (eds. D.A. Holton and J. Seberry) 157-164, Lecture Not. Math. 686, Springer, Berlin, 1978. 
[24] C.D. Godsil, Bounding the diameter of distance regular graphs, Combinatorica 8 (1988), 333-343.

[25] C. D. Godsil, Algebraic Combinatorics, Chapman and Hall, New York, 1993.

[26] J. Gómez, C. Padró, and S. Perennes, Large generalized cycles, Discrete Appl. Math. 89 (1998), no. 1-3, 107-123.

[27] W.H. Haemers, Interlacing eigenvalues and graphs, Linear Algebra Appl. $\mathbf{2 2 7 / 2 2 8}$ (1995), 593-616.

[28] A.J. Hoffman, On the polynomial of a graph, Amer. Math. Monthly 70 (1963), $30-36$.

[29] J.C. Montserrat, "Propiedades Matriciales de los Grafos Dirigidos," Ms. Dissertation (in Spanish), Univ. Politècnica de Catalunya, Spain, 1986.

[30] P. Rowlinson, Linear algebra, in: Graph Connections (ed. L.W. Beineke and R.J. Wilson), Oxford Lecture Ser. Math. Appl., Vol. 5, 86-99, Oxford Univ. Press, New York, 1997.

[31] H. Sachs, Über Teiler, Faktoren und characterische Polynome von Graphen II, Wiss. Z. Techn. Hosch. Ilmenau 13 (1967), 405-412. 